\title{
Cyclic generalized contractions and fixed point results with applications to an integral equation
}

\author{
Hemant Kumar Nashine ${ }^{1}$, Wutiphol Sintunavarat ${ }^{2}$ and Poom Kumam² ${ }^{2 *}$
}

\section{"Correspondence:}

poom.kum@kmutt.ac.th

2Department of Mathematics,

Faculty of Science, King Mongkut's

University of Technology Thonburi (KMUTT), Bangkok, 10140, Thailand

Full list of author information is

available at the end of the article

\begin{abstract}
We set up a new variant of cyclic generalized contractive mappings for a map in a metric space and present existence and uniqueness results of fixed points for such mappings. Our results generalize or improve many existing fixed point theorems in the literature. To illustrate our results, we give some examples. At the same time as applications of the presented theorems, we prove an existence theorem for solutions of a class of nonlinear integral equations.
\end{abstract}

MSC: $47 \mathrm{H} 10 ; 54 \mathrm{H} 25$

Keywords: fixed point; cyclic generalized $(\mathcal{F}, \psi, L)$-contraction; integral equation

\section{Introduction and preliminaries}

All the way through this paper, by $\mathbb{R}^{+}$, we designate the set of all real nonnegative numbers, while $\mathbb{N}$ is the set of all natural numbers.

The celebrated Banach's [1] contraction mapping principle is one of the cornerstones in the development of nonlinear analysis. This principle has been extended and improved in many ways over the years (see, e.g., [2-5]). Fixed point theorems have applications not only in various branches of mathematics but also in economics, chemistry, biology, computer science, engineering, and other fields. In particular, such theorems are used to demonstrate the existence and uniqueness of a solution of differential equations, integral equations, functional equations, partial differential equations, and others. Owing to the magnitude, generalizations of the Banach fixed point theorem have been explored heavily by many authors. This celebrated theorem can be stated as follows.

Theorem 1.1 ([1]) Let $(X, d)$ be a complete metric space and $T$ be a mapping of $X$ into itself satisfying

$$
d(T x, T y) \leq k d(x, y), \quad \forall x, y \in X
$$

where $k$ is a constant in $(0,1)$. Then $T$ has a unique fixed point $x^{*} \in X$.

Inequality (1) implies the continuity of $T$. A natural question is whether we can find contractive conditions which will imply the existence of a fixed point in a complete metric space but will not imply continuity.

( 2012 Nashine et al.; licensee Springer. This is an Open Access article distributed under the terms of the Creative Commons Attribution License (http://creativecommons.org/licenses/by/2.0), which permits unrestricted use, distribution, and reproduction in any medium, provided the original work is properly cited. 
On the other hand, cyclic representations and cyclic contractions were introduced by Kirk et al. [6]. A mapping $T: A \cup B \rightarrow A \cup B$ is called cyclic if $T(A) \subseteq B$ and $T(B) \subseteq A$, where $A, B$ are nonempty subsets of a metric space $(X, d)$. Moreover, $T$ is called a cyclic contraction if there exists $k \in(0,1)$ such that $d(T x, T y) \leq k d(x, y)$ for all $x \in A$ and $y \in B$. Notice that although a contraction is continuous, a cyclic contraction need not to be. This is one of the important gains of this theorem.

Definition 1.1 (See $[6,7])$ Let $(X, d)$ be a metric space. Let $p$ be a positive integer, $A_{1}, A_{2}, \ldots, A_{p}$ be nonempty subsets of $X, Y=\bigcup_{i=1}^{p} A_{i}$, and $T: Y \rightarrow Y$. Then $Y$ is said to be a cyclic representation of $Y$ with respect to $T$ if

(i) $A_{i}, i=1,2, \ldots, p$ are nonempty closed sets, and

(ii) $T\left(A_{1}\right) \subseteq A_{2}, \ldots, T\left(A_{p-1}\right) \subseteq A_{p}, T\left(A_{p}\right) \subseteq A_{1}$.

Following the paper in [6], a number of fixed point theorems on a cyclic representation of $Y$ with respect to a self-mapping $T$ have appeared (see, e.g., [3, 7-15]).

In this paper, we introduce a new class of cyclic generalized $(\mathcal{F}, \psi, L)$-contractive mappings, and then investigate the existence and uniqueness of fixed points for such mappings. Our main result generalizes and improves many existing theorems in the literature. We supply appropriate examples to make obvious the validity of the propositions of our results. To end with, as applications of the presented theorems, we achieve fixed point results for a generalized contraction of integral type and we prove an existence theorem for solutions of a system of integral equations.

\section{Main results}

In this section, we introduce two new notions of a cyclic contraction and establish new results for such mappings.

In the sequel, we fixed the set of functions by $\mathcal{F}, \psi:[0,+\infty) \rightarrow[0,+\infty)$ such that

(i) $\mathcal{F}$ is nondecreasing, continuous, and $\mathcal{F}(0)=0<\mathcal{F}(t)$ for every $t>0$;

(ii) $\psi$ is nondecreasing, right continuous, and $\psi(t)<t$ for every $t>0$.

Define $\mathbf{F}_{1}=\{\mathcal{F}: \mathcal{F}$ satisfies (i) $\}$ and $\Psi_{1}=\{\psi: \psi$ satisfies (ii) $\}$.

We state the notion of a cyclic generalized $(\mathcal{F}, \psi, L)$-contraction as follows.

Definition 2.1 Let $(X, d)$ be a metric space. Let $p$ be a positive integer, $A_{1}, A_{2}, \ldots, A_{p}$ be nonempty subsets of $X$ and $Y=\bigcup_{i=1}^{p} A_{i}$. An operator $T: Y \rightarrow Y$ is said to be a cyclic generalized $(\mathcal{F}, \psi, L)$-contraction for some $\psi \in \Psi_{1}, \mathcal{F} \in \mathbf{F}_{1}$, and $L \geq 0$ if

(a) $Y=\bigcup_{i=1}^{p} A_{i}$ is a cyclic representation of $Y$ with respect to $T$;

(b) for any $(x, y) \in A_{i} \times A_{i+1}, i=1,2, \ldots, p$ (with $A_{p+1}=A_{1}$ ),

$$
\mathcal{F}(d(T x, T y)) \leq \psi(\mathcal{F}(\Theta(x, y)))+L \mathcal{F}\left(\Theta_{1}(x, y)\right)
$$

where

$$
\Theta(x, y)=\max \left\{d(x, y), d(x, T x), d(y, T y), \frac{d(x, T y)+d(y, T x)}{2}\right\}
$$

and

$$
\Theta_{1}(x, y)=\min \{d(x, T x), d(y, T y), d(x, T y), d(y, T x)\} .
$$

Our first main result is the following. 
Theorem 2.1 Let $(X, d)$ be a complete metric space, $p \in \mathbb{N}, A_{1}, A_{2}, \ldots, A_{p}$ be nonempty closed subsets of $X$, and $Y=\bigcup_{i=1}^{p} A_{i}$. Suppose $T: Y \rightarrow Y$ is a cyclic generalized $(\mathcal{F}, \psi, L)$ contraction mapping for some $\psi \in \Psi_{1}$ and $\mathcal{F} \in \mathbf{F}_{1}$. Then $T$ has a unique fixed point. Moreover, the fixed point of $T$ belongs to $\bigcap_{i=1}^{p} A_{i}$.

Proof Let $x_{0} \in A_{1}$ (such a point exists since $A_{1} \neq \emptyset$ ). Define the sequence $\left\{x_{n}\right\}$ in $X$ by

$$
x_{n+1}=T x_{n}, \quad n=0,1,2, \ldots
$$

We shall prove that

$$
\lim _{n \rightarrow \infty} d\left(x_{n}, x_{n+1}\right)=0
$$

If, for some $k$, we have $x_{k+1}=x_{k}$, then (2) follows immediately. So, we can suppose that $d\left(x_{n}, x_{n+1}\right)>0$ for all $n$. From the condition (a), we observe that for all $n$, there exists $i=$ $i(n) \in\{1,2, \ldots, p\}$ such that $\left(x_{n}, x_{n+1}\right) \in A_{i} \times A_{i+1}$. Then, from the condition (b), we have

$$
\mathcal{F}\left(d\left(x_{n}, x_{n+1}\right)\right) \leq \psi\left(\mathcal{F}\left(\Theta\left(x_{n-1}, x_{n}\right)\right)\right)+L \mathcal{F}\left(\Theta_{1}\left(x_{n-1}, x_{n}\right)\right), \quad n=1,2, \ldots
$$

On the other hand, we have

$$
\begin{aligned}
\Theta\left(x_{n-1}, x_{n}\right) & =\max \left\{d\left(x_{n-1}, x_{n}\right), d\left(x_{n+1}, x_{n}\right), \frac{1}{2} d\left(x_{n-1}, x_{n+1}\right)\right\} \\
& =\max \left\{d\left(x_{n-1}, x_{n}\right), d\left(x_{n}, x_{n+1}\right)\right\}
\end{aligned}
$$

and

$$
\Theta_{1}\left(x_{n-1}, x_{n}\right)=\min \left\{d\left(x_{n-1}, x_{n}\right), d\left(x_{n}, x_{n+1}\right), d\left(x_{n-1}, x_{n+1}\right), d\left(x_{n}, x_{n}\right)\right\}=0 .
$$

Suppose that $\max \left\{d\left(x_{k-1}, x_{k}\right), d\left(x_{k}, x_{k+1}\right)\right\}=d\left(x_{k}, x_{k+1}\right)$ for some $k \in \mathbb{N}$. Then $\Theta\left(x_{k-1}, x_{k}\right)=$ $d\left(x_{k}, x_{k+1}\right)$, so

$$
\mathcal{F}\left(d\left(x_{k}, x_{k+1}\right)\right) \leq \psi\left(\mathcal{F}\left(d\left(x_{k}, x_{k+1}\right)\right)\right)<\mathcal{F}\left(d\left(x_{k}, x_{k+1}\right)\right),
$$

a contradiction. Hence,

$$
\Theta\left(x_{n-1}, x_{n}\right)=d\left(x_{n-1}, x_{n}\right)
$$

and thus

$$
\mathcal{F}\left(d\left(x_{n}, x_{n+1}\right)\right) \leq \psi\left(\mathcal{F}\left(d\left(x_{n-1}, x_{n}\right)\right)\right)<\mathcal{F}\left(d\left(x_{n-1}, x_{n}\right)\right)
$$

Similarly, we have

$$
\mathcal{F}\left(d\left(x_{n-1}, x_{n}\right)\right)<\mathcal{F}\left(d\left(x_{n-2}, x_{n-1}\right)\right) .
$$


Thus, from (4) and (5), we get

$$
\mathcal{F}\left(d\left(x_{n+1}, x_{n}\right)\right)<\mathcal{F}\left(d\left(x_{n}, x_{n-1}\right)\right)
$$

for all $n \in \mathbb{N}$. Now, from

$$
\mathcal{F}\left(d\left(x_{n+1}, x_{n}\right)\right) \leq \psi\left(\mathcal{F}\left(d\left(x_{n}, x_{n-1}\right)\right)\right)<\cdots<\psi^{n}\left(\mathcal{F}\left(d\left(x_{1}, x_{0}\right)\right)\right)
$$

and the property of $\psi$, we obtain $\lim _{n \rightarrow \infty} \mathcal{F}\left(d\left(x_{n+1}, x_{n}\right)\right)=0$, and consequently (2) holds.

Now, we shall prove that $\left\{x_{n}\right\}$ is a Cauchy sequence in $(X, d)$. Suppose, on the contrary, that $\left\{x_{n}\right\}$ is not a Cauchy sequence. Then there exists $\varepsilon>0$ for which we can find two sequences of positive integers $\{m(k)\}$ and $\{n(k)\}$ such that for all positive integers $k$,

$$
m(k)>n(k) \geq k, \quad d\left(x_{m(k)}, x_{n(k)}\right) \geq \varepsilon
$$

Further, corresponding to $n(k)$, we can choose $m(k)$ in such a way that it is the smallest integer with $m(k)>n(k) \geq k$ satisfying (6). Then we have

$$
d\left(x_{m(k)-1}, x_{n(k)}\right)<\varepsilon .
$$

Using (6), (7), and the triangular inequality, we get

$$
\begin{aligned}
\varepsilon & \leq d\left(x_{n(k)}, x_{m(k)}\right) \\
& \leq d\left(x_{n(k)}, x_{m(k)-1}\right)+d\left(x_{m(k)-1}, x_{m(k)}\right) \\
& <\varepsilon+d\left(x_{m(k)-1}, x_{m(k)}\right)
\end{aligned}
$$

Thus, we have

$$
\varepsilon \leq d\left(x_{n(k)}, x_{m(k)}\right)<\varepsilon+d\left(x_{m(k)-1}, x_{m(k)}\right) .
$$

Passing to the limit as $k \rightarrow \infty$ in the above inequality and using (2), we obtain

$$
\lim _{k \rightarrow \infty} d\left(x_{n(k)}, x_{m(k)}\right)=\varepsilon^{+}
$$

On the other hand, for all $k$, there exists $j(k) \in\{1, \ldots, p\}$ such that $n(k)-m(k)+j(k) \equiv 1[p]$. Then $x_{m(k)-j(k)}$ (for $k$ large enough, $\left.m(k)>j(k)\right)$ and $x_{n(k)}$ lie in different adjacently labeled sets $A_{i}$ and $A_{i+1}$ for certain $i \in\{1, \ldots, p\}$. Using (b), we obtain

$$
\mathcal{F}\left(d\left(x_{m(k)-j(k)+1}, x_{n(k)+1}\right)\right) \leq \psi\left(\mathcal{F}\left(\Theta\left(x_{m(k)-j(k)}, x_{n(k)}\right)\right)\right)+L \mathcal{F}\left(\Theta_{1}\left(x_{m(k)-j(k)}, x_{n(k)}\right)\right)
$$

for all $k$. Now, we have

$$
\begin{aligned}
\Theta\left(x_{m(k)-j(k)}, x_{n(k)}\right)= & \max \left\{d\left(x_{m(k)-j(k)}, x_{n(k)}\right), d\left(x_{m(k)-j(k)+1}, x_{m(k)-j(k)}\right), d\left(x_{n(k)+1}, x_{n(k)}\right),\right. \\
& \left.\frac{d\left(x_{m(k)-j(k)}, x_{n(k)+1}\right)+d\left(x_{n(k)}, x_{m(k)-j(k)+1}\right)}{2}\right\},
\end{aligned}
$$


and

$$
\begin{aligned}
\Theta_{1}\left(x_{m(k)-j(k)}, x_{n(k)}\right)= & \min \left\{d\left(x_{m(k)-j(k)+1}, x_{m(k)-j(k)}\right), d\left(x_{n(k)+1}, x_{n(k)}\right),\right. \\
& \left.d\left(x_{m(k)-j(k)}, x_{n(k)+1}\right), d\left(x_{n(k)}, x_{m(k)-j(k)+1}\right)\right\}
\end{aligned}
$$

for all $k$. Using the triangular inequality, we get

$$
\begin{aligned}
& \left|d\left(x_{m(k)-j(k)}, x_{n(k)}\right)-d\left(x_{n(k)}, x_{m(k)}\right)\right| \\
& \quad \leq d\left(x_{\left.m(k)-j(k), x_{m(k)}\right)}\right. \\
& \quad \leq \sum_{l=0}^{j(k)-1} d\left(x_{m(k)-j(k)+l}, x_{m(k)-j(k)+l+1}\right) \\
& \quad \leq \sum_{l=0}^{p-1} d\left(x_{m(k)-j(k)+l}, x_{m(k)-j(k)+l+1}\right) \rightarrow 0 \quad \text { as } k \rightarrow \infty(\text { from }(2)),
\end{aligned}
$$

which implies from (8) that

$$
\lim _{k \rightarrow \infty} d\left(x_{m(k)-j(k)}, x_{n(k)}\right)=\varepsilon
$$

Using (2), we have

$$
\lim _{k \rightarrow \infty} d\left(x_{m(k)-j(k)+1}, x_{m(k)-j(k)}\right)=0
$$

and

$$
\lim _{k \rightarrow \infty} d\left(x_{n(k)+1}, x_{n(k)}\right)=0 .
$$

Again, using the triangular inequality, we get

$$
\left|d\left(x_{m(k)-j(k)}, x_{n(k)+1}\right)-d\left(x_{m(k)-j(k)}, x_{n(k)}\right)\right| \leq d\left(x_{n(k)}, x_{n(k)+1}\right) .
$$

Passing to the limit as $k \rightarrow \infty$ in the above inequality, using (14) and (12), we get

$$
\lim _{k \rightarrow \infty} d\left(x_{m(k)-j(k)}, x_{n(k)+1}\right)=\varepsilon
$$

Similarly, we have

$$
\left|d\left(x_{n(k)}, x_{m(k)-j(k)+1}\right)-d\left(x_{m(k)-j(k)}, x_{n(k)}\right)\right| \leq d\left(x_{m(k)-j(k)}, x_{m(k)-j(k)+1}\right) .
$$

Passing to the limit as $k \rightarrow \infty$, using (2) and (12), we obtain

$$
\lim _{k \rightarrow \infty} d\left(x_{n(k)}, x_{m(k)-j(k)+1}\right)=\varepsilon
$$

Similarly, we have

$$
\lim _{k \rightarrow \infty} d\left(x_{m(k)-j(k)+1}, x_{n(k)+1}\right)=\varepsilon .
$$


Now, it follows from (12)-(16) and the continuity of $\varphi$ that

$$
\lim _{k \rightarrow \infty} \Theta\left(x_{m(k)-j(k)}, x_{n(k)}\right)=\max \{\varepsilon, 0\}=\varepsilon
$$

and

$$
\lim _{k \rightarrow \infty} \Theta_{1}\left(x_{m(k)-j(k)}, x_{n(k)}\right)=\min \{0,0, \varepsilon, \varepsilon\}=0 .
$$

Passing to the limit as $k \rightarrow \infty$ in (9), using (17), (18), (19), and the condition (ii), we obtain

$$
\mathcal{F}(\varepsilon) \leq \psi(\mathcal{F}(\varepsilon))+L \cdot 0<\mathcal{F}(\varepsilon)
$$

which is a contradiction. Thus, we proved that $\left\{x_{n}\right\}$ is a Cauchy sequence in $(X, d)$.

Since $(X, d)$ is complete, there exists $x^{*} \in X$ such that

$$
\lim _{n \rightarrow \infty} x_{n}=x^{*}
$$

We shall prove that

$$
x^{*} \in \bigcap_{i=1}^{p} A_{i}
$$

From the condition (a), and since $x_{0} \in A_{1}$, we have $\left\{x_{n p}\right\}_{n \geq 0} \subseteq A_{1}$. Since $A_{1}$ is closed, from (20), we get that $x^{*} \in A_{1}$. Again, from the condition (a), we have $\left\{x_{n p+1}\right\}_{n \geq 0} \subseteq A_{2}$. Since $A_{2}$ is closed, from (20), we get that $x^{*} \in A_{2}$. Continuing this process, we obtain (21).

Now, we shall prove that $x^{*}$ is a fixed point of $T$. Indeed, from (21), since for all $n$ there exists $i(n) \in\{1,2, \ldots, p\}$ such that $x_{n} \in A_{i(n)}$, applying (b) with $x=x^{*}$ and $y=x_{n}$, we obtain

$$
\mathcal{F}\left(d\left(T x^{*}, x_{n+1}\right)\right)=\mathcal{F}\left(d\left(T x^{*}, T x_{n}\right)\right) \leq \psi\left(\mathcal{F}\left(\Theta\left(x^{*}, x_{n}\right)\right)\right)+L \mathcal{F}\left(\Theta_{1}\left(x^{*}, x_{n}\right)\right)
$$

for all $n$. On the other hand, we have

$$
\Theta\left(x^{*}, x_{n}\right)=\max \left\{d\left(x^{*}, x_{n}\right), d\left(x^{*}, T x^{*}\right), d\left(x_{n}, x_{n+1}\right), \frac{d\left(x^{*}, x_{n+1}\right)+d\left(x_{n}, T x^{*}\right)}{2}\right\}
$$

and

$$
\Theta_{1}\left(x^{*}, x_{n}\right)=\min \left\{d\left(x^{*}, T x^{*}\right), d\left(x_{n}, x_{n+1}\right), d\left(x^{*}, x_{n+1}\right), d\left(x_{n}, T x^{*}\right)\right\} .
$$

Passing to the limit as $n \rightarrow \infty$ in the above inequality and using (20), we obtain that

$$
\lim _{n \rightarrow \infty} \Theta\left(x^{*}, x_{n}\right)=\max \left\{d\left(x^{*}, T x^{*}\right), \frac{1}{2} d\left(x^{*}, T x^{*}\right)\right\} \quad \text { and } \quad \lim _{n \rightarrow \infty} \Theta_{1}\left(x^{*}, x_{n}\right)=0
$$

Passing to the limit as $n \rightarrow \infty$ in (22), using (23) and (20), we get

$$
\mathcal{F}\left(d\left(x^{*}, T x^{*}\right)\right) \leq \psi\left(\mathcal{F}\left(\max \left\{d\left(x^{*}, T x^{*}\right), \frac{1}{2} d\left(x^{*}, T x^{*}\right)\right\}\right)\right) .
$$


Suppose that $d\left(x^{*}, T x^{*}\right)>0$. In this case, we have

$$
\max \left\{d\left(x^{*}, T x^{*}\right), \frac{d\left(x^{*}, T x^{*}\right)}{2}\right\}=d\left(x^{*}, T x^{*}\right),
$$

which implies that

$$
\mathcal{F}\left(d\left(x^{*}, T x^{*}\right)\right) \leq \psi\left(\mathcal{F}\left(d\left(x^{*}, T x^{*}\right)\right)\right)<\mathcal{F}\left(d\left(x^{*}, T x^{*}\right)\right),
$$

a contradiction. Then we have $d\left(x^{*}, T x^{*}\right)=0$, that is, $x^{*}$ is a fixed point of $T$.

Finally, we prove that $x^{\prime \prime}$ is the unique fixed point of $T$. Assume that $y^{\prime \prime}$ is another fixed point of $T$, that is, $T y^{*}=y^{*}$. From the condition (a), this implies that $y^{*} \in \bigcap_{i=1}^{p} A_{i}$. Then we can apply (b) for $x=x^{*}$ and $y=y^{*}$. We obtain

$$
\mathcal{F}\left(d\left(x^{*}, y^{*}\right)\right)=\mathcal{F}\left(d\left(T x^{*}, T y^{*}\right)\right) \leq \psi\left(\mathcal{F}\left(\Theta\left(x^{*}, y^{*}\right)\right)\right)+L \mathcal{F}\left(\Theta_{1}\left(x^{*}, y^{*}\right)\right) .
$$

Since $x^{*}$ and $y^{*}$ are fixed points of $T$, we can show easily that $\Theta\left(x^{*}, y^{*}\right)=d\left(x^{*}, y^{*}\right)$ and $\Theta_{1}\left(x^{*}, y^{*}\right)=0$. If $d\left(x^{*}, y^{*}\right)>0$, we get

$$
\begin{aligned}
\mathcal{F}\left(d\left(x^{*}, y^{\prime \prime}\right)\right) & =\mathcal{F}\left(d\left(T x^{*}, T y^{*}\right)\right) \leq \psi\left(\mathcal{F}\left(\Theta\left(x^{*}, y^{*}\right)\right)\right) \\
& =\psi\left(\mathcal{F}\left(d\left(x^{*}, y^{\prime \prime}\right)\right)\right)<\mathcal{F}\left(d\left(x^{*}, y^{*}\right)\right),
\end{aligned}
$$

a contradiction. Then we have $d\left(x^{*}, y^{*}\right)=0$, that is, $x^{*}=y^{*}$. Thus, we proved the uniqueness of the fixed point.

In the following, we deduce some fixed point theorems from our main result given by Theorem 2.1.

If we take $p=1$ and $A_{1}=X$ in Theorem 2.1, then we get immediately the following fixed point theorem.

Corollary 2.1 Let $(X, d)$ be a complete metric space and $T: X \rightarrow X$ satisfy the following condition: there exist $\psi \in \Psi_{1}, \mathcal{F} \in \mathbf{F}_{1}$, and $L \geq 0$ such that

$$
\begin{aligned}
\mathcal{F}(d(T x, T y)) \leq & \psi\left(\mathcal{F}\left(\max \left\{d(x, y), d(T x, x), d(y, T y), \frac{d(x, T y)+d(y, T x)}{2}\right\}\right)\right) \\
& +L \mathcal{F}(\min \{d(x, y), d(T x, x), d(y, T y), d(x, T y), d(y, T x)\})
\end{aligned}
$$

for all $x, y \in X$. Then $T$ has a unique fixed point.

Remark 2.1 Corollary 2.1 extends and generalizes many existing fixed point theorems in the literature $[1,16-21]$.

Corollary 2.2 Let $(X, d)$ be a complete metric space, $p \in \mathbb{N}, A_{1}, A_{2}, \ldots, A_{p}$ be nonempty closed subsets of $X, Y=\bigcup_{i=1}^{p} A_{i}$, and $T: Y \rightarrow Y$. Suppose that there exist $\psi \in \Psi_{1}$ and $\mathcal{F} \in \mathbf{F}_{1}$ such that

(a') $Y=\bigcup_{i=1}^{p} A_{i}$ is a cyclic representation of $Y$ with respect to $T$; 
(b') for any $(x, y) \in A_{i} \times A_{i+1}, i=1,2, \ldots, p\left(\right.$ with $\left.A_{p+1}=A_{1}\right)$,

$$
\mathcal{F}(d(T x, T y)) \leq \psi(\mathcal{F}(d(x, y)))
$$

Then $T$ has a unique fixed point. Moreover, the fixed point of $T$ belongs to $\bigcap_{i=1}^{p} A_{i}$.

Remark 2.2 Corollary 2.2 is similar to Theorem 2.1 in [7].

Remark 2.3 Taking in Corollary $2.2 \psi(t)=k t$ with $k \in(0,1)$, we obtain a generalized version of Theorem 1.3 in [6].

Corollary 2.3 Let $(X, d)$ be a complete metric space, $p \in \mathbb{N}, A_{1}, A_{2}, \ldots, A_{p}$ be nonempty closed subsets of $X, Y=\bigcup_{i=1}^{p} A_{i}$, and $T: Y \rightarrow Y$. Suppose that there exist $\psi \in \Psi_{1}$ and $\mathcal{F} \in \mathbf{F}_{1}$ such that

(a') $Y=\bigcup_{i=1}^{p} A_{i}$ is a cyclic representation of $Y$ with respect to $T$;

(b') for any $(x, y) \in A_{i} \times A_{i+1}, i=1,2, \ldots, p$ (with $\left.A_{p+1}=A_{1}\right)$,

$$
\mathcal{F}(d(T x, T y)) \leq \psi\left(\mathcal{F}\left(\frac{d(x, T y)+d(y, T x)}{2}\right)\right)
$$

Then $T$ has a unique fixed point. Moreover, the fixed point of $T$ belongs to $\bigcap_{i=1}^{p} A_{i}$.

Remark 2.4 Taking in Corollary $2.3 \psi(t)=k t$ with $k \in(0,1)$, we obtain a generalized version of Theorem 3 in [13].

Corollary 2.4 Let $(X, d)$ be a complete metric space, $p \in \mathbb{N}, A_{1}, A_{2}, \ldots, A_{p}$ be nonempty closed subsets of $X, Y=\bigcup_{i=1}^{p} A_{i}$, and $T: Y \rightarrow Y$. Suppose that there exist $\psi \in \Psi_{1}$ and $\mathcal{F} \in \mathbf{F}_{1}$ such that

(a') $Y=\bigcup_{i=1}^{p} A_{i}$ is a cyclic representation of $Y$ with respect to $T$;

(b') for any $(x, y) \in A_{i} \times A_{i+1}, i=1,2, \ldots, p$ (with $\left.A_{p+1}=A_{1}\right)$,

$$
\mathcal{F}(d(T x, T y)) \leq \psi(\mathcal{F}(\max \{d(x, T x), d(y, T y)\})) .
$$

Then $T$ has a unique fixed point. Moreover, the fixed point of $T$ belongs to $\bigcap_{i=1}^{p} A_{i}$.

Remark 2.5 Taking in Corollary 2.4 $\psi(t)=k t$ with $k \in(0,1)$, we obtain a generalized version of Theorem 5 in [13].

Corollary 2.5 Let $(X, d)$ be a complete metric space, $p \in \mathbb{N}, A_{1}, A_{2}, \ldots, A_{p}$ be nonempty closed subsets of $X, Y=\bigcup_{i=1}^{p} A_{i}$, and $T: Y \rightarrow Y$. Suppose that there exist $\psi \in \Psi_{1}$ and $\mathcal{F} \in \mathbf{F}_{1}$ such that

(a) $Y=\bigcup_{i=1}^{p} A_{i}$ is a cyclic representation of $Y$ with respect to $T$;

(b) for any $(x, y) \in A_{i} \times A_{i+1}, i=1,2, \ldots, p$ (with $\left.A_{p+1}=A_{1}\right)$,

$$
\mathcal{F}(d(T x, T y)) \leq \psi(\mathcal{F}(\max \{d(x, y), d(x, T x), d(y, T y)\}))
$$

Then $T$ has a unique fixed point. Moreover, the fixed point of $T$ belongs to $\bigcap_{i=1}^{p} A_{i}$. 
We provide some examples to illustrate our obtained Theorem 2.1.

Example 2.1 Let $X=\mathbb{R}$ with the usual metric. Suppose $A_{1}=[-1,0]$ and $A_{2}=[0,1]$ and $Y=\bigcup_{i=1}^{2} A_{i}$. Define $T: Y \rightarrow Y$ such that $T x=\frac{-x}{3}$ for all $x \in Y$. It is clear that $\bigcup_{i=1}^{2} A_{i}$ is a cyclic representation of $Y$ with respect to $T$. Let $\psi \in \Psi_{1}$ be defined by $\psi(t)=\frac{t}{2}$ and $\mathcal{F} \in \mathbf{F}_{1}$ of the form $\mathcal{F}(t)=k t, k>0$. For all $x, y \in Y$ and $L \geq 0$, we have

$$
\begin{aligned}
\mathcal{F}(d(T x, T y)) & =k d(T x, T y) \\
& =\frac{k|x-y|}{3} \\
& \leq \frac{k|x-y|}{2} \\
& \leq \frac{k(\Theta(x, y))}{2} \\
& \leq \frac{k(\Theta(x, y))}{2}+\operatorname{Lk} \Theta_{1}(x, y) \\
& =\psi(\mathcal{F}(\Theta(x, y)))+L \mathcal{F}\left(\Theta_{1}(x, y)\right) .
\end{aligned}
$$

So, $T$ is a cyclic generalized $(\mathcal{F}, \psi, L)$-contraction for any $L \geq 0$. Therefore, all conditions of Theorem 2.1 are satisfied $(p=2)$, and so $T$ has a unique fixed point (which is $x^{*}=0 \in$ $\left.\bigcap_{i=1}^{2} A_{i}\right)$.

Example 2.2 Let $X=\mathbb{R}$ with the usual metric. Suppose $A_{1}=[-\pi / 2,0]$ and $A_{2}=[0, \pi / 2]$ and $Y=\bigcup_{i=1}^{2} A_{i}$. Define the mapping $T: Y \rightarrow Y$ by

$$
T x= \begin{cases}-\frac{1}{3} x|\cos (1 / x)| & \text { if } x \in[-\pi / 2,0) \cup(0, \pi / 2], \\ 0 & \text { if } x=0 .\end{cases}
$$

Clearly, we have $T\left(A_{1}\right) \subseteq A_{2}$ and $T\left(A_{2}\right) \subseteq A_{1}$. Moreover, $A_{1}$ and $A_{2}$ are nonempty closed subsets of $X$. Therefore, $\bigcup_{i=1}^{2} A_{i}$ is a cyclic representation of $Y$ with respect to $T$.

Now, let $(x, y) \in A_{1} \times A_{2}$ with $x \neq 0$ and $y \neq 0$, we have

$$
\begin{aligned}
d(T x, T y) & =|T x-T y| \\
& =\left|\frac{1}{3} x\right| \cos (1 / x)\left|+\frac{1}{3} y\right| \cos (1 / y)|| \\
& =\frac{1}{3}|| x|| \cos (1 / x)|+| y|| \cos (1 / y)|| \\
& \leq \frac{1}{3}(|x|+|y|) .
\end{aligned}
$$

On the other hand, we have

$$
|x|=-x \leq-x+\frac{1}{3}|x \cos (1 / x)|=-x-\frac{1}{3} x|\cos (1 / x)| \leq\left|x+\frac{1}{3} x\right| \cos (1 / x)||=d(x, T x)
$$

and

$$
|y|=y \leq y+\frac{1}{3}|y \cos (1 / y)|=\left|y+\frac{1}{3} y\right| \cos (1 / y)||=d(y, T y) .
$$


Then we have

$$
\begin{aligned}
d(T x, T y) & \leq \frac{2}{3} \max \{d(x, T x), d(y, T y)\} \\
& \leq \frac{2}{3} \max \left\{d(x, y), d(x, T x), d(y, T y),\left(\frac{d(x, T y)+d(y, T x)}{2}\right)\right\} .
\end{aligned}
$$

Define the function $\psi:[0, \infty) \rightarrow[0, \infty)$ by $\psi(t)=\frac{2 t}{3}$, for all $t \geq 0$ and $\mathcal{F} \in \mathbf{F}_{1}$ of the form $\mathcal{F}(t)=k t, k>0$ and $L \geq 0$. Then we have

$$
\mathcal{F}(d(T x, T y)) \leq \psi(\mathcal{F}(\Theta(x, y)))+L \mathcal{F}(\Theta(x, y)) .
$$

Moreover, we can show that (24) holds if $x=0$ or $y=0$. Similarly, we also get (24) holds for $(x, y) \in A_{2} \times A_{1}$.

Now, all the conditions of Theorem 2.1 are satisfied (with $p=2$ ), we deduce that $T$ has a unique fixed point $x^{*} \in A_{1} \cap A_{2}=\{0\}$.

\section{An application to an integral equation}

In this section, we apply the result given by Theorem 2.1 to study the existence and uniqueness of solutions to a class of nonlinear integral equations.

We consider the nonlinear integral equation

$$
u(t)=\int_{0}^{T} G(t, s) f(s, u(s)) d s \quad \text { for all } t \in[0, T]
$$

where $T>0, f:[0, T] \times \mathbb{R} \rightarrow \mathbb{R}$ and $G:[0, T] \times[0, T] \rightarrow[0, \infty)$ are continuous functions.

Let $X=C([0, T])$ be the set of real continuous functions on $[0, T]$. We endow $X$ with the standard metric

$$
d_{\infty}(u, v)=\max _{t \in[0, T]}|u(t)-v(t)| \quad \text { for all } u, v \in X .
$$

It is well known that $\left(X, d_{\infty}\right)$ is a complete metric space.

Let $(\alpha, \beta) \in X^{2},\left(\alpha_{0}, \beta_{0}\right) \in \mathbb{R}^{2}$ such that

$$
\alpha_{0} \leq \alpha(t) \leq \beta(t) \leq \beta_{0} \quad \text { for all } t \in[0, T] .
$$

We suppose that for all $t \in[0, T]$, we have

$$
\alpha(t) \leq \int_{0}^{T} G(t, s) f(s, \beta(s)) d s
$$

and

$$
\beta(t) \geq \int_{0}^{T} G(t, s) f(s, \alpha(s)) d s .
$$

We suppose that for all $s \in[0, T], f(s, \cdot)$ is a decreasing function, that is,

$$
x, y \in \mathbb{R}, \quad x \geq y \quad \Longrightarrow \quad f(s, x) \leq f(s, y) .
$$


We suppose that

$$
\sup _{t \in[0, T]} \int_{0}^{T} G(t, s) d s \leq 1 .
$$

Finally, we suppose that, for all $s \in[0, T]$, for all $x, y \in \mathbb{R}$ with $\left(x \leq \beta_{0}\right.$ and $\left.y \geq \alpha_{0}\right)$ or $\left(x \geq \alpha_{0}\right.$ and $\left.y \leq \beta_{0}\right)$,

$$
\begin{aligned}
|f(s, x)-f(s, y)| \leq & \psi\left(\max \left\{|x-y|,|x-T x|,|y-T y|, \frac{|x-T y|+|y-T x|}{2}\right\}\right) \\
& +L \min \{|x-T x|,|y-T y|,|x-T y|,|y-T x|\},
\end{aligned}
$$

where $\psi:[0, \infty) \rightarrow[0, \infty)$ is a nondecreasing function that belongs to $\Psi_{1}$ and $L \geq 0$.

Now, define the set

$$
\mathcal{C}=\{u \in C([0, T]): \alpha \leq u(t) \leq \beta \text { for all } t \in[0, T]\} .
$$

We have the following result.

Theorem 3.1 Under the assumptions (26)-(31), problem (25) has one and only one solution $u^{*} \in \mathcal{C}$

Proof Define the closed subsets of $X, A_{1}$ and $A_{2}$, by

$$
A_{1}=\{u \in X: u \leq \beta\}
$$

and

$$
A_{2}=\{u \in X: u \geq \alpha\} .
$$

Define the mapping $T: X \rightarrow X$ by

$$
T u(t)=\int_{0}^{T} G(t, s) f(s, u(s)) d s \quad \text { for all } t \in[0, T] .
$$

We shall prove that

$$
T\left(A_{1}\right) \subseteq A_{2} \quad \text { and } \quad T\left(A_{2}\right) \subseteq A_{1} .
$$

Let $u \in A_{1}$, that is,

$$
u(s) \leq \beta(s) \text { for all } s \in[0, T] .
$$

Using condition (29), since $G(t, s) \geq 0$ for all $t, s \in[0, T]$, we obtain that

$$
G(t, s) f(s, u(s)) \geq G(t, s) f(s, \beta(s)) \quad \text { for all } t, s \in[0, T] .
$$


The above inequality with condition (27) implies that

$$
\int_{0}^{T} G(t, s) f(s, u(s)) d s \geq \int_{0}^{T} G(t, s) f(s, \beta(s)) d s \geq \alpha(t)
$$

for all $t \in[0, T]$. Then we have $T u \in A_{2}$.

Similarly, let $u \in A_{2}$, that is,

$$
u(s) \geq \alpha(s) \quad \text { for all } s \in[0, T] .
$$

Using condition (29), since $G(t, s) \geq 0$ for all $t, s \in[0, T]$, we obtain that

$$
G(t, s) f(s, u(s)) \leq G(t, s) f(s, \alpha(s)) \quad \text { for all } t, s \in[0, T] \text {. }
$$

The above inequality with condition (28) implies that

$$
\int_{0}^{T} G(t, s) f(s, u(s)) d s \leq \int_{0}^{T} G(t, s) f(s, \alpha(s)) d s \leq \beta(t)
$$

for all $t \in[0, T]$. Then we have $T u \in A_{1}$. Finally, we deduce that (32) holds.

Now, let $(u, v) \in A_{1} \times A_{2}$, that is, for all $t \in[0, T]$,

$$
u(t) \leq \beta(t), \quad v(t) \geq \alpha(t) .
$$

This implies, from condition (26), that for all $t \in[0, T]$,

$$
u(t) \leq \beta_{0}, \quad v(t) \geq \alpha_{0} .
$$

Now, using conditions (30) and (31), we can write that for all $t \in[0, T]$, we have

$$
\begin{array}{rl}
\mid T u & T v \mid(t) \\
\leq & \int_{0}^{T} G(t, s)|f(s, u(s))-f(s, v(s))| d s \\
\leq & \int_{0}^{T} G(t, s) \psi(\max \{|u(s)-v(s)|,|u(s)-T u(s)|,|v(s)-T v(s)|, \\
& \left.\left.\frac{|u(s)-T v(s)|+|v(s)-T u(s)|}{2}\right\}\right) d s \\
& +\int_{0}^{T} G(t, s) L \min \{|u(s)-T u(s)|,|v(s)-T v(s)|,|u(s)-T v(s)|,|v(s)-T u(s)|\} d s \\
\leq & \psi\left(\max \left\{d_{\infty}(u, v), d_{\infty}(u, T u), d_{\infty}(v, T v), \frac{d_{\infty}(u, T v)+d_{\infty}(v, T u)}{2}\right\}\right) \int_{0}^{T} G(t, s) d s \\
& +L \min \left\{d_{\infty}(u, T u), d_{\infty}(v, T v), d_{\infty}(u, T v), d_{\infty}(v, T u)\right\} \int_{0}^{T} G(t, s) d s \\
\leq & \psi\left(\max \left\{d_{\infty}(u, v), d_{\infty}(u, T u), d_{\infty}(v, T v), \frac{d_{\infty}(u, T v)+d_{\infty}(v, T u)}{2}\right\}\right) \\
& +L \min \left\{d_{\infty}(u, T u), d_{\infty}(v, T v), d_{\infty}(u, T v), d_{\infty}(v, T u)\right\} .
\end{array}
$$


This implies that

$$
\mathcal{F}\left(d_{\infty}(T u, T v)\right) \leq \psi(\mathcal{F}(\Theta(u, v)))+L \mathcal{F}\left(\Theta_{1}(u, v)\right)
$$

where $\mathcal{F} \in \mathbf{F}_{1}$ of the form $\mathcal{F}(t)=t$. Using the same technique, we can show that the above inequality holds also if we take $(u, v) \in A_{2} \times A_{1}$.

Now, all the conditions of Theorem 2.1 are satisfied (with $p=2$ ), we deduce that $T$ has a unique fixed point $u^{*} \in A_{1} \cap A_{2}=\mathcal{C}$, that is, $u^{*} \in \mathcal{C}$ is the unique solution to (25).

\section{Competing interests}

The authors declare that they have no competing interests.

\section{Authors' contributions}

All authors contributed equally and significantly in writing this paper. All authors read and approved the final manuscript.

\section{Author details}

'Department of Mathematics, Disha Institute of Management and Technology, Satya Vihar, Vidhansabha-Chandrakhuri Marg, Mandir Hasaud, Raipur, Chhattisgarh 492101, India. ${ }^{2}$ Department of Mathematics, Faculty of Science, King Mongkut's University of Technology Thonburi (KMUTT), Bangkok, 10140, Thailand.

\section{Acknowledgements}

The second author would like to thank the Research Professional Development Project under the Science Achievement Scholarship of Thailand (SAST). Moreover, the third author was supported by the Commission on Higher Education (CHE), the Thailand Research Fund (TRF) and the King Mongkut's University of Technology Thonburi (KMUTT) (Grant No. MRG5580213)

Received: 13 June 2012 Accepted: 12 November 2012 Published: 28 November 2012

\section{References}

1. Banach, S: Sur les opérations dans les ensembles abstraits et leur application aux equations itegrales. Fundam. Math. 3, 133-181 (1922)

2. Chauhan, S, Sintunavarat, W, Kumam, P: Common fixed point theorems for weakly compatible mappings in fuzzy metric spaces using (JCLR) property. Appl. Math. 3(9), 976-982 (2012)

3. Pacurar, M: Fixed point theory for cyclic Berinde operators. Fixed Point Theory 12(2), 419-428 (2011)

4. Sintunavarat, W, Cho, YJ, Kumam, P: Common fixed point theorems for c-distance in ordered cone metric spaces. Comput. Math. Appl. 62, 1969-1978 (2011)

5. Sintunavarat, W, Kumam, P: Generalized common fixed point theorems in complex valued metric spaces and applications. J. Inequal. Appl. 2012,84 (2012)

6. Kirk, WA, Srinivasan, PS, Veeramani, P: Fixed points for mappings satisfying cyclical contractive conditions. Fixed Point Theory 4(1), 79-89 (2003)

7. Pacurar, M, Rus, IA: Fixed point theory for cyclic $\varphi$-contractions. Nonlinear Anal. 72, 1181-1187 (2010)

8. Agarwal, RP, Alghamdi, MA, Shahzad, N: Fixed point theory for cyclic generalized contractions in partial metric spaces. Fixed Point Theory Appl. 2012, 40 (2012)

9. Aydi, H, Vetro, C, Sintunavarat, W, Kumam, P: Coincidence and fixed points for contractions and cyclical contractions in partial metric spaces. Fixed Point Theory Appl. 2012, 124 (2012)

10. Karapınar, E: Fixed point theory for cyclic weak $\phi$-contraction. Appl. Math. Lett. 24(6), 822-825 (2011)

11. Karapınar, E, Sadaranagni, K: Fixed point theory for cyclic $(\phi-\psi)$-contractions. Fixed Point Theory Appl. 2011, 69 (2011)

12. Mongkolkeha, C, Kumam, P: Best proximity point theorems for generalized cyclic contractions in ordered metric spaces. J. Optim. Theory Appl. 155, 215-226 (2012)

13. Petric, MA: Some results concerning cyclical contractive mappings. Gen. Math. 18(4), 213-226 (2010)

14. Rus, IA: Cyclic representations and fixed points. Ann. T. Popoviciu Semin. Funct. Equ. Approx. Convexity 3, 171-178 (2005)

15. Sintunavarat, W, Kumam, P: Common fixed point theorem for cyclic generalized multi-valued contraction mappings. Appl. Math. Lett. 25(11), 1849-1855 (2012)

16. Bianchini, RMT: Su un problema di S. Reich riguardante la teoria dei punti fissi. Boll. Unione Mat. Ital., Ser. IV 5, 103-108 (1972)

17. Boyd, DW, Wong, JS: On nonlinear contractions. Proc. Am. Math. Soc. 20, 458-469 (1969)

18. Chatterjea, SK: Fixed point theorems. C. R. Acad. Bulgare Sci. 25, 727-730 (1972)

19. Hardy, GE, Rogers, TD: A generalization of a fixed point theorem of Reich. Can. Math. Bull. 16(2), $201-206$ (1973)

20. Kannan, R: Some results on fixed points. Bull. Calcutta Math. Soc. 10, $71-76$ (1968)

21. Reich, S: Some remarks concerning contraction mappings. Can. Math. Bull. 14, 121-124 (1971)

doi:10.1186/1687-1812-2012-217

Cite this article as: Nashine et al.: Cyclic generalized contractions and fixed point results with applications to an integral equation. Fixed Point Theory and Applications 2012 2012:217. 